\title{
D0 results on diphoton direct production and double parton interactions in photon +3 jet events
}

\author{
Lee Sawyer ${ }^{* i t+}$ \\ Louisiana Tech University \\ E-mail: sawyer@latech.edu
}

\begin{abstract}
We report the measurement of differential diphoton direct production cross sections and a study of photon +3 -jet events with double parton (DP) interactions, based on data taken with the DØ experiment at the Fermilab Tevatron proton-antiproton collider.

We measure single differential cross sections as a function of the diphoton mass, the transverse momentum of the diphoton system, the azimuthal angle between the photons, and the polar scattering angle of the photons. In addition, we measure double differential cross sections considering the last three kinematic variables in three diphoton mass bins. The results are compared with different perturbative $\mathrm{QCD}$ predictions and event generators.

We have used a sample of photon +3 -jet events collected by the $D \emptyset$ experiment with an integrated luminosity of about $1 \mathrm{fb}^{-1}$ to determine the fraction of events with double parton scattering $\left(f_{D P}\right)$ in a single $p \bar{p}$ collision at $\sqrt{s}=1.96 \mathrm{TeV}$. The DP fraction and effective cross section $\left(\sigma_{e f f}\right)$, a process-independent scale parameter related to the parton density inside the nucleon, are measured in three intervals of the second (ordered in $p_{T}$ ) jet transverse momentum $p_{T}^{\text {jet } 2}$ within the range $15<p_{T}^{\text {jet } 2}<30 \mathrm{GeV}$. In this range, $f_{D P}$ varies between $0.23<f_{D P}<0.47$, while $\sigma_{\text {eff }}$ has the average value $\sigma_{\text {eff }}^{\text {ave }}=16.4 \pm 0.3$ (stat) \pm 2.3 (syst) $\mathrm{mb}$.
\end{abstract}

XVIII International Workshop on Deep-Inelastic Scattering and Related Subjects

April 19 -23, 2010

Convitto della Calza, Firenze, Italy

\footnotetext{
${ }^{*}$ Speaker.

${ }^{\dagger}$ My thanks to the DØ QCD physics group conveners (Don Lincoln, Dmitry Bandurin, Sabine Lammers) for their help preparing this talk.

*Delivered via teleconference from a seaside hotel in Barcelona, due to the Eyjafjallajökull volcano eruptions. This may be a new paradigm for $21^{s t}$ century conferences: fly $3 / 4$ of the way to the conference, find a nice place to stay, and phone it in.
} 


\section{Introduction}

Direct photon production in hadron collisions provides a precise probe of perturbative Quantum Chromodynamics (pQCD), particularly as a test of soft gluon resummation. Diphoton production can proceed through Born-level and box diagrams in $\mathrm{pQCD}$, as well as single or double parton fragmentation. In addition, high mass diphoton pairs form a major irreducible background to searches for Higgs boson production via the $H \rightarrow \gamma \gamma$ decay channel. Therefore, the measurement of diphoton production cross sections at the Tevatron collider is important for both comparisons to QCD as well as for search for the Higgs or new phenomena producing diphoton resonances. In this note we present single differential cross-section for diphoton production, in bins of the diphoton invariant mass $M_{\gamma \gamma}$, total transverse momentum of the pair $p_{T}^{\gamma \gamma}$, and polar scattering angle $\left|\cos \theta^{*}\right|$. We also present the first double differential cross sections measured at the Tevatron.

Photon and multijet events can also be used as a probe of the partonic structure of the protons and antiprotons. Of particular interest is the use of those events as a way to differentiate doubleparton interactions in a single $p \bar{p}$ collision. We present measurements of the fraction of doubleparton interaction, using events tagged with a high transverse momentum photon, with at least three associated jets in the events. The rate of such double-parton events is related to the dynamics distribution of the initial state partons.

The data presented were taken in $p \bar{p}$ collisions at a center-of-mass energy of $\sqrt{s}=1.96 \mathrm{TeV}$, collected with detailed description of the D0 detector can be found in Ref. [1]. We select events with photons with calorimeter and track isolations and also satisfying shower shape cuts based on the calorirmeter and CPS. Please see details in [2] and [3]. Jets are defined by the Run II midpoint cone jet algorithm [4] with a cone radius of $R_{\text {cone }}=\sqrt{(\Delta y)^{2}+(\Delta \phi)^{2}}=0.7$ in rapidity $y$ and azimuthal angle $\phi$.

\section{Direct Diphoton Production}

The data used in this analysis were collected using a combination of triggers requiring at least two clusters of energy in the EM calorimeter with loose shower shape requirements and varying $p_{T}$ thresholds between $15 \mathrm{GeV}$ and $25 \mathrm{GeV}$, and correspond to an integrated luminosity of $4.2 \pm$ $0.3 \mathrm{fb}^{-1}$. The two photons had to have $p_{T}>21$ and $20 \mathrm{GeV}$ and be with $|\eta|<0.9$ and a separation of $\Delta R(\gamma \gamma)>0.4$, and satisfy $M_{\gamma \gamma}>p_{T}^{\gamma \gamma}$.

Cross sections were calculated as

$$
\frac{d \sigma}{d X}=\frac{N_{\gamma \gamma}}{\varepsilon \times A \times L \times \Delta_{X}}
$$

where $X$ represents the kinematical variable being studies $\left(M_{\gamma \gamma}, p_{T}^{\gamma \gamma}, \Delta \phi^{\gamma \gamma},\left|\cos \theta^{*}\right|\right)$ and $\Delta_{X}$ is the bin width in this variable. The event selection efficiency $\varepsilon$, acceptance $A$, and integrated $L$, along with the background estimation on the number of diphoton events $N_{\gamma \gamma}$ contribute a total systemic uncertainty of around $18 \%$, relatively flat over most of the $M_{\gamma \gamma}$ range.

Single differential cross section are shown in Figures 1,1,2, and 2. The exerperimental measurements are compared to theoretical predictions based on the RESBOS [5] and DIPHOX[6] Monte Carlos. Each were run with the CTEQ6.6M [7] PDFs with $\mu_{R}=\mu_{F}=M_{\gamma \gamma}$. Comparisons 
are also made to the PYTHIA [8] Monte Carlo (v6.420) using the CTEQ5L [9] PDFs and parameter set "Tune A" [10].
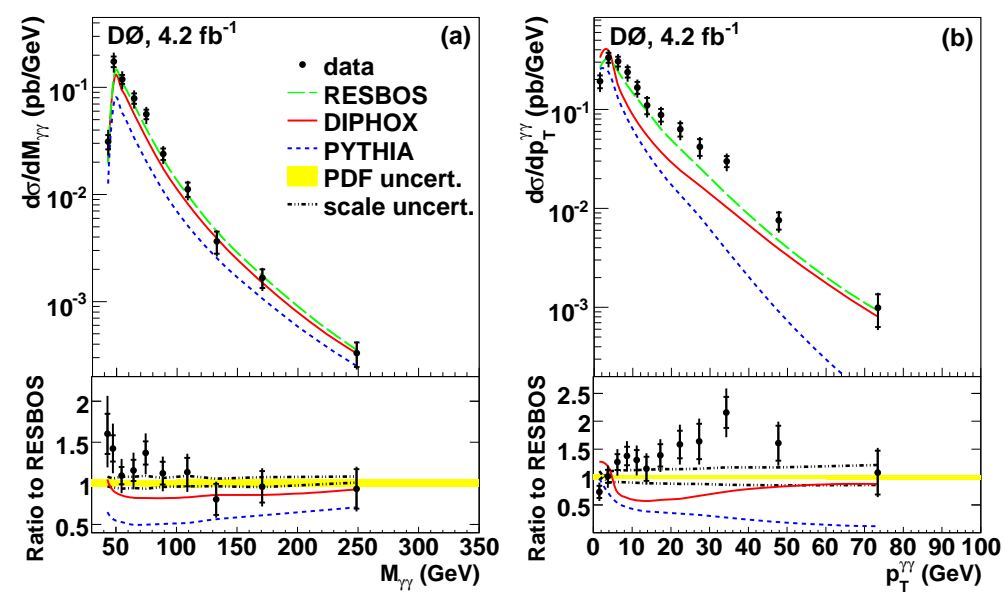

Figure 1: The diphoton cross section as a funtion of the invariant mass $M_{\gamma \gamma}$ of the photon pair. The diphoton cross section as a function of the transverse momentum $p_{T}^{\gamma \gamma}$ of the photon pair. Error bars include systematic uncertainties for both plots.
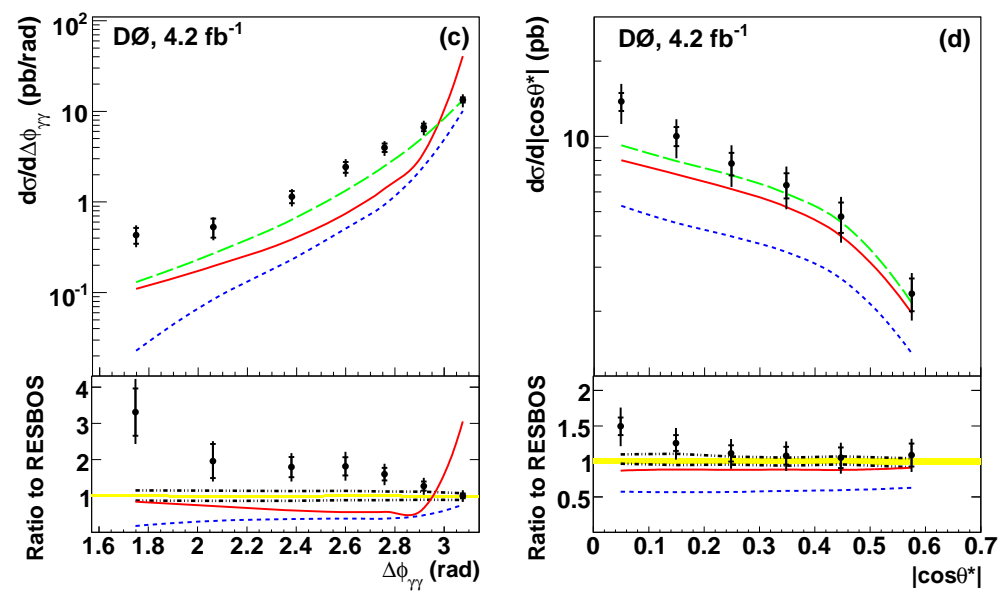

Figure 2: The diphoton cross section as a function of the azimuthal opening angle $\Delta \phi^{\gamma \gamma}$ of the photon pair. The diphoton cross section as a function of the scattering angle $\left|\cos \theta^{*}\right|$ of the photon pair. Error bars include systematic uncertainties for both plots.

Double differential cross section are formed in three bins of the diphoton invariant mass: $30 \leq$ $M_{\gamma \gamma}<50 \mathrm{GeV}, 50 \leq M_{\gamma \gamma}<80 \mathrm{GeV}$, and $80 \leq M_{\gamma \gamma}<350 \mathrm{GeV}$. Figure 3 shows the the cross sections for $p_{T}^{\gamma \gamma}, \Delta \phi^{\gamma \gamma}$ and $\left|\cos \theta^{*}\right|$ in the three mass bins.

\section{Double Parton Events}

Many features of high energy inelastic hadron collisions depend directly on the parton structure of hadrons. The inelastic scattering of nucleons need not to occur only through a single parton- 

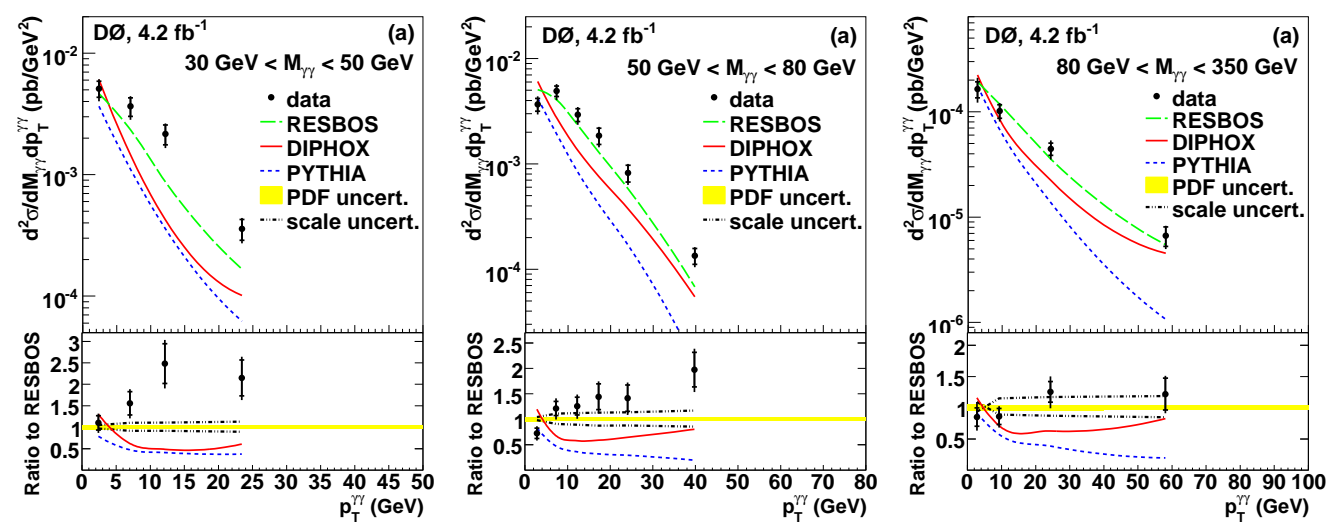

Figure 3: The diphoton cross section as a function of the transverse momentum $p_{T}^{\gamma \gamma}$ of the photon pair, in the three bins of the invariant mass $M_{\gamma \gamma}$ of the photon pair described in the text. Error bars include systematic uncertainties.

parton interaction and the contribution from double parton (DP) collisions can be significant. The rate of events with multiple parton scattering depends on how the partons are spatially distributed within the nucleon. The DP cross sections can be expressed as [11][14] $\sigma_{D P}=\frac{\sigma_{A} \sigma_{B}}{\sigma_{e f f}}$, where $\sigma_{A}$ and $\sigma_{B}$ are the cross sections of two independent partonic scatterings $\mathrm{A}$ and $\mathrm{B}$. The process independent scaling parameter $\sigma_{e f f}$ has the units of cross section. Its relation to the spatial distribution of partons within the proton has been discussed in [11] through [14]. The ratio $\sigma_{B} / \sigma_{\text {eff }}$ can be interpreted as the probability for partonic process B to occur provided that process A has already occurred.

We have measured the rate of DP events, using the data collected with the D0 detector during Run IIa, which after applying all the data quality criteria and the trigger selections, corresponds to an integrated luminosity of about $1.02 \pm 0.06 \mathrm{fb}^{-1}$. To determine the fraction of DP events, we select the sample of $\gamma+3$ jets events with the requirement of only one event vertex ("1VTX" sample). The event vertex should have at least three associated tracks and the distance to the center of the detector along the beam axis should be less than $60 \mathrm{~cm}$. There should be at least one photon candidate with $p_{T}^{\gamma}$ between 60 and $80 \mathrm{GeV}$ and passing quality cuts, and at least 3 jets with $p_{T}^{\text {jet }}>25 / 15 \mathrm{GeV}$ (leading/second or third) and $|y|<3.0$ and passing jet quality requirements.

The $p_{T}$ spectrum for jets from dijet events falls faster than that for jets resulting from initial or final state radiation in the $\gamma+$ jet events, and thus DP fractions should depend on the jet $p_{T}$ [11][14]. The DP fractions and $\sigma_{\text {eff }}$ are determined in three $p_{T}^{\text {jet } 2}$ bins $15-20,20-25$, and 25-30 GeV using a data driven method that exploits a difference in distributions of the $\Delta S$ variable; defined as $\Delta S=$ $\Delta \phi\left(p_{T}^{\gamma, j e t_{i}}, p_{T}^{j e t_{j}, j e t_{k}}\right)$, representing the opening angle of the two best balancing pairs of jets. The value of $\Delta S$ is chosen so that it minimizes on of the three variables $S_{p_{T}}, S_{p_{T}^{\prime}}$, and $S_{\phi}$, based on jet $p_{T}$ resolution, average $p_{T}$, and $\phi$ resolution, respectively [3]. In most events, $\Delta S$ is minimized by pairing the photon with the leading jet.

In figure 4 we show the measured fraction of DP $\left(f_{D P}\right)$ events, in three intervals of $p_{T}^{\text {jet2 }}$. These are extracted from the $\Delta S_{\phi}, \Delta S_{p_{T}}$ and $\Delta S_{p_{T}^{\prime}}$ as indicated in the figure. On the right, a comparison to the $f_{D P}$ measurement is made to the PYTHIA Monte Carlo with two common tunes, tune $\mathrm{A}$ and 
Perugia tune S0 [15].
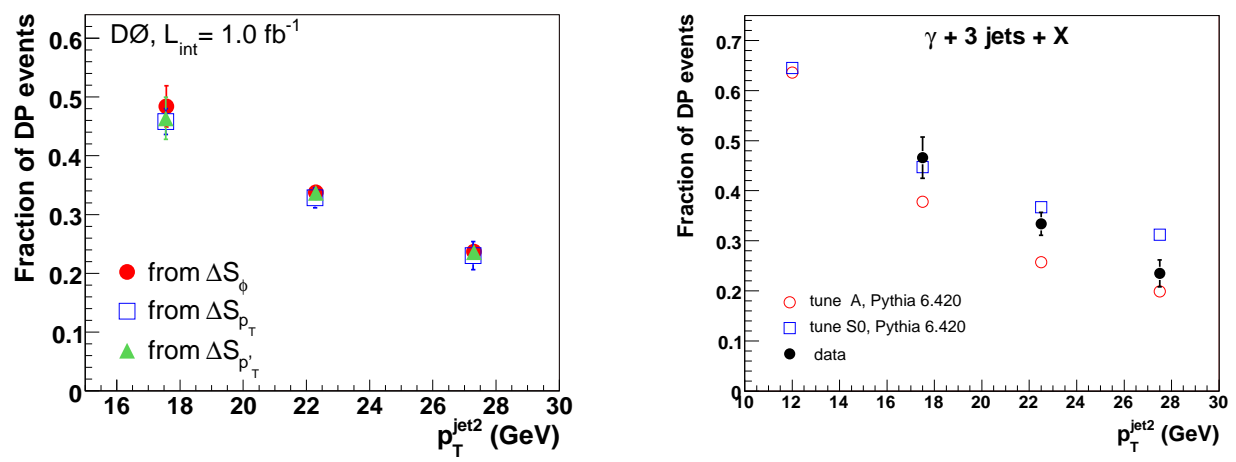

Figure 4: a) The fraction of double parton (DP) measured in three $p_{T}^{\text {jet } 2}$ intervals, extracted from the $\Delta S_{\phi}$, $\Delta S_{p_{T}}$ and $\Delta S_{p_{T}^{\prime}}$ distributions described in the text. b) A comparison of the average measured fraction of DP events in the three $p_{T}^{\text {jet } 2}$ intervals, compared to prediction based on the PYTHIA Monte Carlo

The fraction of DP events, combined with a similar measurement of double interaction (DI) events, is used to extract the effective cross section $\sigma_{e f f}$. Figure 5 shows the value of $\sigma_{e f f}$ measured in the three bins of $p_{T}^{\text {jet } 2}$. Only a weak $p_{T}$ dependence is observed. The value averaged over the three bins is $\sigma_{e f f}^{\text {ave }}=16.4 \pm 0.3$ (stat) \pm 2.3 (syst) $\mathrm{mb}$.

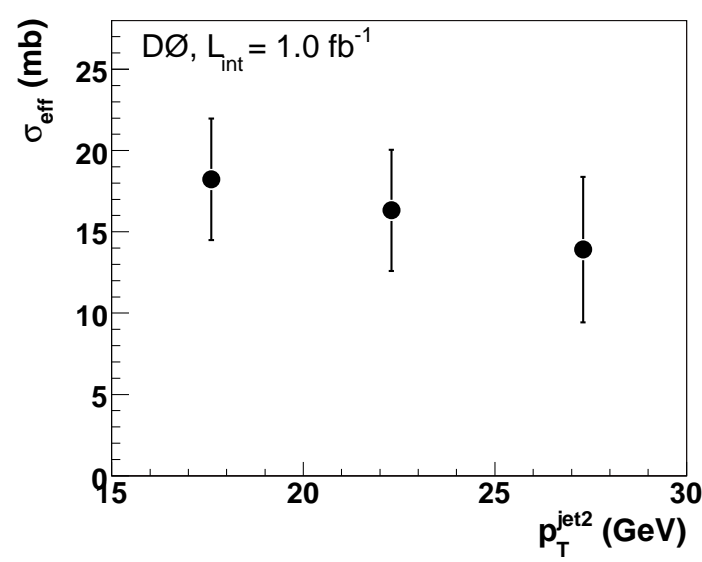

Figure 5: The effective cross section $\sigma_{e f f}$ for double parton (DP) events, as measured in three $p_{T}^{j e t 2}$ intervals.

\section{Summary}

We have measured single- and (for the first time) double-differential cross sections for direct diphoton production in $p \bar{p}$ collisions at $\sqrt{s}=1.96 \mathrm{TeV}$ with $4.2 \mathrm{fb}^{-1}$ of integrated luminosity. Measurements are compared to state-of-art theoretical predictions such as DIPHOX and RESBOS, as well as PYTHIA. None of the theoretical predictions fully describes the data in all kinematic regions of the four variables considered. 
We have also measured fraction of Double Parton events in three $p_{T}$ bins of the second jet : 15-20, 20-25,25-30 GeV. The fraction $f_{D P}$ varies from about 0.47 at $15-20 \mathrm{GeV}$ to 0.22 at $25-$ $30 \mathrm{GeV}$. A first measurement of the process-independent effective cross section $\sqrt{s}=1.96 \mathrm{TeV}$, which defines the rate of Double Parton events, is measured in the same jet $p_{T}$ bins with an average value of $16.4 \pm 0.3$ (stat) \pm 2.3 (syst) mb. Double Parton production can be a significant background to many rare processes, especially those with multi-jet final states.

\section{References}

[1] V. M. Abazov et al. (D0 Collaboration), Nucl. Instrum. Methods Phys. Res. A 565, 463 (2006).

[2] V. M. Abazov et al. (D0 Collaboration), Phys. Lett. B 690, 108 (2010).

[3] V. M. Abazov et al. (D0 Collaboration), Phys. Rev. D 81, 052012 (2010).

[4] G. C. Blazey et al., in Proceedings of the Workshop: QCD and Weak Boson Physics in Run II, edited by U. Baur, R.K. Ellis, and D. Zeppenfeld, (Batavia, Illinois, 2000) p. 47, see Section 3.5.

[5] C. Balazs, E.L. Berger, S. Mrenna, and C.-P. Yuan, Phys. Rev. D 57, 6934 (1998); C. Balazs, E.L. Berger, P. Nadolsky, and C.-P. Yuan, Phys. Rev. D 76, 013009 (2007).

[6] T. Binoth, J.-Ph. Guillet, E. Pilon, and M. Werlen, Eur. Phys. J. C 16, 311 (2000).

[7] W.K. Tung et al., JHEP 0702, 052 (2007).

[8] T. Sjöstrand et al., Comput. Phys. Commun. 135, 238 (2001).

[9] H. L. Lai et al. (CTEQ Collaboration), Eur. Phys. J. C 12, 375 (2000).

[10] R. D. Field (CDF Collaboration), in the Proceedings of APS / DPF / DPB Summer Study on the Future of Particle Physics (Snowmass 2001), Snowmass, Colorado, 30 Jun - 21 Jul 2001, pp P501

[11] P. V. Landshoff and J. C. Polkinghorne, Phys. rev. D 18, 3344 (1978).

[12] C. Goebel, F. Halzen, and D. M. Scott, Phys. Rev. D 22, 2789 (1980).

[13] N. Paver and D. Trelani, Nuevo Cimento A 70, 215 (1982); L. Ametlier, N. Paver, and D. Trelani, Phys Lett. B 169, 289 (1986).

[14] B. Humpert, Phys. Lett. B 131, 461 (1983); B. Humpert and R. Odorico, Phys. Lett. B 154, 211 (1985).

[15] T. Sjostrand and P. Z. Skands, Eur. Phys. J. C 39 (2005) 129 